01

\title{
Математическое моделирование двумерной диодной системы с полевым эмиттером лезвийной формы
}

\author{
(C) Е.М. Виноградова, Г.Г. Доронин, Н.В. Егоров
}

Санкт-Петербургский государственный университет, 198504 Санкт-Петербург, Россия

e-mail: vincat2008@yandex.ru

Поступило в Редакцию 27 марта 2019 г.

В окончательной редакции 6 сентября 2019 г.

Принято к публикации 1 ноября 2019 г.

Представлено вычисление распределения электростатического потенциала в двумерной диодной эмиссионной системе с полевым эмиттером лезвийной формы на плоской подложке. Анод — плоскость, параллельная подложке. Для вычисления распределения потенциала влияние эмиттера заменено влиянием заряженной нити, так что нулевая эквипотенциаль представляет собой поверхность эмиттера. Для решения граничной задачи использован метод разделения переменных в декартовых координатах. Все геометрические размеры системы являются параметрами задачи.

Ключевые слова: микро- и наноэлектроника, полевой эмиттер, математическое моделирование, электростатический потенциал, граничная задача.

DOI: $10.21883 /$ JTF.2020.04.49075.140-19

\section{Введение}

Электронно-вакуумные устройства на основе полевой электронной эмиссии широко используются в различных областях науки и техники. Полевые эмиттеры привлекают внимание исследователей благодаря своим уникальным эмиссионным свойствам и потенциальным технологическим применением [1-05]. Однако, как правило, одиночные полевые острия позволяют получить небольшие значения эмиссионного тока [6-8]. Увеличение тока возможно за счет увеличения площади эмиссии, когда в качестве полевого катода используются либо многоострийная система, либо эмиттер лезвийной формы с острым краем. Края слоев двухмерных полупроводниковых наноматериалов, например, графена, гафния, также обладают высокой эффективностью эмиссии и могут рассматриваться как полевые эмиттеры [9-13]. Построение адекватных математических моделей для полевых эмиссионных систем, а так же их численная реализация позволяют правильно интерпретировать имеющиеся данные [14-16].

В настоящей работе представлено моделирование диодной эмиссионной системы с полевым катодом лезвийной формы на плоской подложке. Анод представляет собой плоскость, параллельную подложке. Для нахождения распределения электростатического потенциала во всей области системы используется метод разделения переменных в декартовых координатах. На рис. 1 представлено трехмерное схематическое изображение исследуемой системы. Так как длина катода по переменной $z$ не ограничена, то распределение потенциала $U(x, y)$ зависит от двух координат $(x, y)$ и не зависит от $z$. Потенциалы катода и подложки полагаются равными нулю. Рассмотрим решение задачи с произвольными граничными условиями первого рода на остальных границах диодной системы.

\section{Математическая модель}

Поскольку любую эквипотенциальную поверхность можно считать виртуальным катодом, то, для того чтобы вычислить электростатический потенциал во всей области диодной системы, влияние полевого эмиттера на распределение поля можно заменить влиянием заряженной нити с плотностью зарядов $\tau(y)$, расположенной в плоскости $x O y$ с координатами $x=x_{0}, \quad 0 \leq y \leq y_{0}$ (рис. 1). Будем считать, что форму реального эмиттера задает нулевая эквипотенциаль [17-19].

Параметры системы: $y=0\left(0 \leq x \leq x_{1}\right)$ - поверхность подложки эмиттера, $y=y_{1}\left(0 \leq x \leq x_{1}\right)-$ поверхность анода, $x=0, x=x_{1}\left(0 \leq y \leq y_{1}\right)$ - границы области по переменной $x, y=L$ - высота эмиттера.

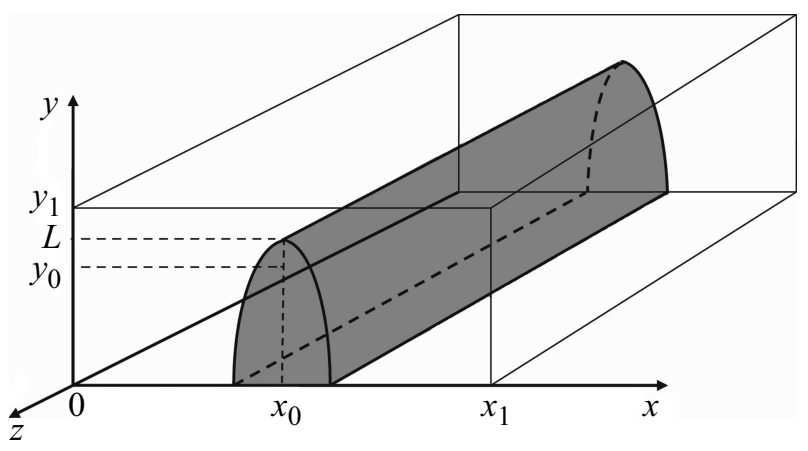

Рис. 1. Схематическое изображение диодной эмиссионной системы. 
Функция распределения электростатического потенциала $U(x, y)$ удовлетворяет уравнению Пуассона и граничным условиям:

$$
\left\{\begin{array}{l}
\frac{\partial^{2} U(x, y)}{\partial x^{2}}+\frac{\partial^{2} U(x, y)}{\partial y^{2}}=-\frac{1}{\varepsilon_{0}} \rho(x, y) \\
0 \leq x \leq x_{1}, \quad 0 \leq y \leq y_{1} \\
U(x, 0)=0, \quad 0 \leq x \leq x_{1} \\
U(0, y)=f_{1}(y), \quad 0 \leq y \leq y_{1} \\
U\left(x, y_{1}\right)=f_{2}(x), \quad 0 \leq x \leq x_{1} \\
U\left(x_{1}, y\right)=f_{3}(y), \quad 0 \leq y \leq y_{1}
\end{array}\right.
$$

Функцию $\rho(x, y)$, входящую в правую часть уравнения Пуассона $(1)$, определим через плотность заряженной нити $\tau(y)$, порождающую ненулевое пространственное распределение заряда $\rho(y)$ вблизи нити [19].

\section{Решение граничной задачи}

Решение исходной граничной задачи (1) $U(x, y)$ можно представить в виде суммы решений двух граничных задач для функций $U_{1}(x, y)$ и $U_{2}(x, y)$ :

$$
\begin{gathered}
U(x, y)=U_{1}(x, y)+U_{2}(x, y), \\
\left\{\begin{array}{l}
\frac{\partial^{2} U_{1}(x, y)}{\partial x^{2}}+\frac{\partial^{2} U_{1}(x, y)}{\partial y^{2}}=0, \\
0 \leq x \leq x_{1}, \quad 0 \leq y \leq y_{1}, \\
U_{1}(x, 0)=0, \quad 0 \leq x \leq x_{1}, \\
U_{1}(0, y)=f_{1}(y), \quad 0 \leq y \leq y_{1}, \\
U_{1}\left(x, y_{1}\right)=f_{2}(x), \quad 0 \leq x \leq x_{1}, \\
U_{1}\left(x_{1}, y\right)=f_{3}(y), \quad 0 \leq y \leq y_{1}, \\
\frac{\partial^{2} U_{2}(x, y)}{\partial x^{2}}+\frac{\partial^{2} U_{2}(x, y)}{\partial y^{2}}=-\frac{1}{\varepsilon_{0}} \rho(x, y), \\
0 \leq x \leq x_{1}, \quad 0 \leq y \leq y_{1}, \\
U_{2}(x, 0)=0, \quad 0 \leq x \leq x_{1}, \\
U_{2}(0, y)=0, \quad 0 \leq y \leq y_{1}, \\
U_{2}\left(x, y_{1}\right)=0, \quad 0 \leq x \leq x_{1}, \\
U_{2}\left(x_{1}, y\right)=0, \quad 0 \leq y \leq y_{1},
\end{array}\right.
\end{gathered}
$$

где $U_{1}(x, y)$ - решение граничной задачи для уравнения Лапласа с заданными граничными условиями (1), отвечающее за распределение потенциала, создаваемого системой электродов, и $U_{2}(x, y)-$ решение граничной задачи для уравнения Пуассона с однородными граничными условиями, отвечающее за поле, создаваемое заряженной нитью.
Решение граничной задачи (3) имеет вид

$$
\begin{aligned}
& U_{1}(x, y)=\sum_{m=1}^{\infty} a_{m} \frac{\operatorname{sh}\left[\alpha_{m} y\right]}{\operatorname{sh}\left[\alpha_{m} y_{1}\right]} \sin \alpha_{m} x \\
& +\sum_{n=1}^{\infty}\left(b_{n} \frac{\operatorname{sh}\left[\beta_{n}\left(x_{1}-x\right)\right]}{\operatorname{sh}\left[\beta_{n} x_{1}\right]}+c_{n} \frac{\operatorname{sh}\left[\beta_{n} x\right]}{\operatorname{sh}\left[\beta_{n} x_{1}\right]}\right) \sin \beta_{n} y .
\end{aligned}
$$

Собственные значения $\alpha_{m}$ и $\beta_{n}$ вычисляются по формуле

$$
\alpha_{m}=\pi m / x_{1}, \quad \beta_{n}=\pi n / y_{1},
$$

$a_{m}, b_{n}, c_{n}-$ коэффициенты разложения в ряд Фурье граничных функций:

$$
\begin{gathered}
a_{m}=\frac{2}{x_{1}} \int_{0}^{x_{1}} f_{2}(x) \sin \alpha_{m} x d x, \quad b_{n}=\frac{2}{y_{1}} \int_{0}^{y_{1}} f_{1}(y) \sin \beta_{n} y d y, \\
c_{n}=\frac{2}{y_{1}} \int_{0}^{y_{1}} f_{3}(y) \sin \beta_{n} y d y .
\end{gathered}
$$

Распределение потенциала $U_{2}(x, y)$ как решение граничной задачи (4) можно представить в виде [19] при $0 \leq y \leq y_{0}$

$$
\begin{aligned}
& U_{2}(x, y)=\sum_{m=1}^{\infty} \frac{2 \sin \alpha_{m} x_{0}}{\pi m \varepsilon_{0}}\left[\frac{\operatorname{sh}\left[\alpha_{m}\left(y_{1}-y\right)\right]}{\operatorname{sh}\left[\alpha_{m} y_{1}\right]}\right. \\
& \times \int_{0}^{y} \tau(\eta) \operatorname{sh}\left[\alpha_{m} \eta\right] d \eta-\frac{\operatorname{sh}\left[\alpha_{m} y\right]}{\operatorname{sh}\left[\alpha_{m} y_{1}\right]} \\
& \left.\times \int_{y}^{y_{0}} \tau(\eta) \operatorname{sh}\left[\alpha_{m}\left(\eta-y_{1}\right)\right] d \eta\right] \sin \alpha_{m} x,
\end{aligned}
$$

при $y_{0} \leq y \leq y_{1}$

$$
\begin{aligned}
U_{2}(x, y)= & \sum_{m=1}^{\infty} \frac{2 \sin \alpha_{m} x_{0}}{\pi m \varepsilon_{0}} \frac{\operatorname{sh}\left[\alpha_{m}\left(y_{1}-y\right)\right]}{\operatorname{sh}\left[\alpha_{m} y_{1}\right]} \\
& \times \int_{0}^{y_{0}} \tau(\eta) \operatorname{sh}\left[\alpha_{m} \eta\right] d \eta \sin \alpha_{m} x .
\end{aligned}
$$

Таким образом, формулы (2),(5)-(9) дают аналитическое решение граничной задачи (1) в любой точке области моделируемой эмиссионной диодной системы.

\section{Результаты численных расчетов}

Для расчета распределения потенциала $U(x, y)$ (2) рассмотрен случай, когда функция плотности зарядов принимает постоянное значение $\tau(y)=\tau=$ const, a функции на границах области задают однородное поле:

$$
f_{1}(y)=U_{0} y / y_{1}, \quad f_{2}(x)=U_{0}, \quad f_{3}(y)=U_{0} y / y_{1} .
$$




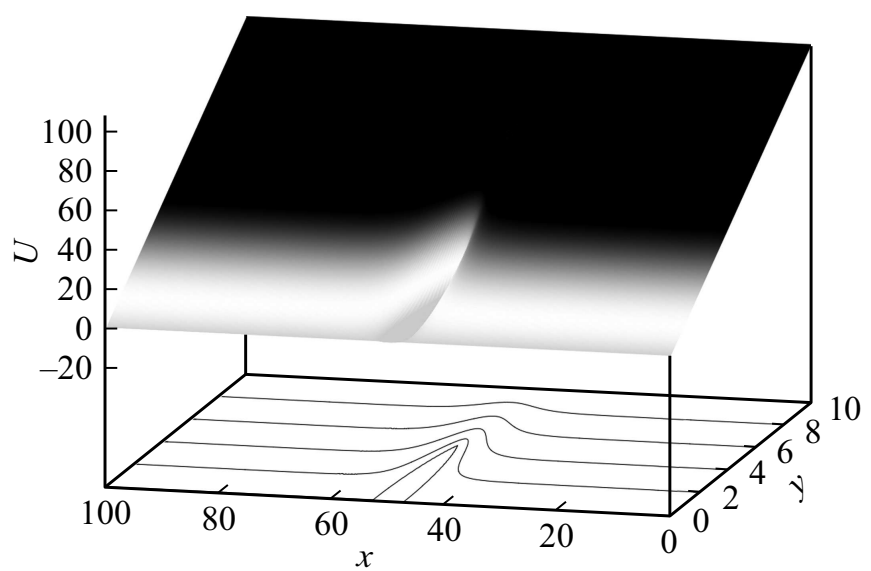

Рис. 2. Распределения электростатического потенциала и эквипотенциальных линий при $\tau=-50$.

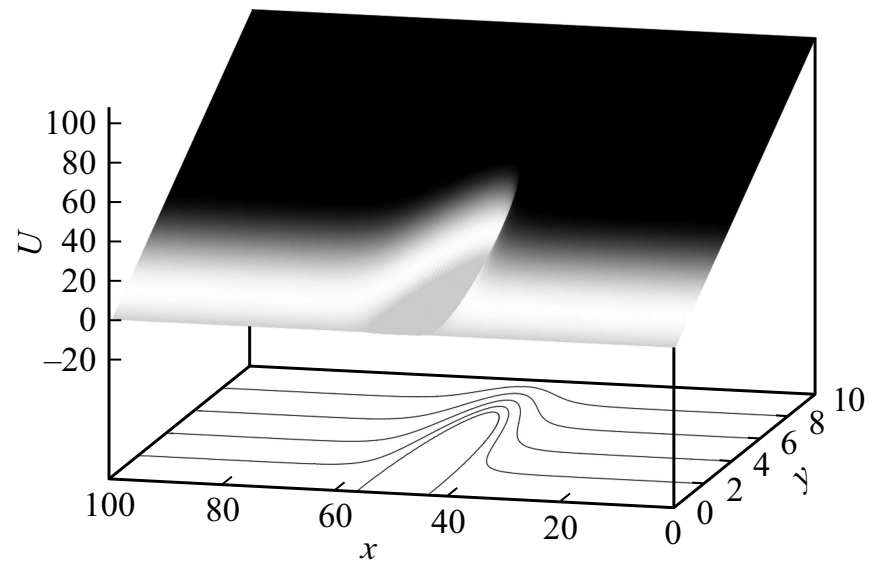

Рис. 3. Распределения электростатического потенциала и эквипотенциальных линий при $\tau=-200$.

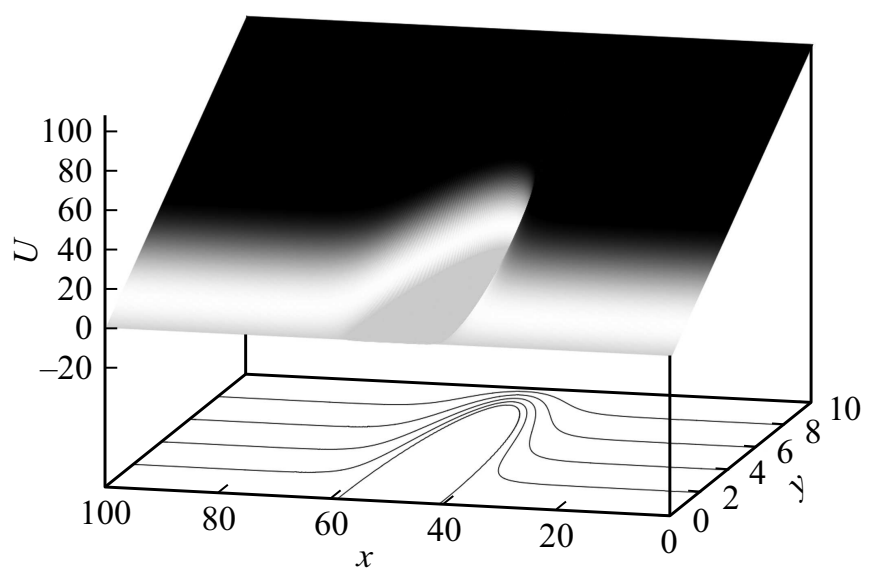

Рис. 4. Распределения электростатического потенциала и эквипотенциальных линий при $\tau=-500$.
Тогда решение граничной задачи (1) имеет вид при $0 \leq y \leq y_{0}$

$$
\begin{aligned}
& U(x, y)=U_{0} \frac{y}{y_{1}} \\
& +\tau \sum_{m=1}^{\infty} \frac{2 \sin \alpha_{m} x_{0}}{\alpha_{m} \pi m \varepsilon_{0}}\left[\frac{\operatorname{sh}\left[\alpha_{m}\left(y_{1}-y\right)\right]}{\operatorname{sh}\left[\alpha_{m} y_{1}\right]}\left(\operatorname{ch}\left[\alpha_{m} y\right]-1\right)\right. \\
& +\frac{\operatorname{sh}\left[\alpha_{m} y\right]}{\operatorname{sh}\left[\alpha_{m} y_{1}\right]}\left(\operatorname{ch}\left[\alpha_{m}\left(y_{1}-y\right)\right]-\operatorname{ch}\left[\alpha_{m}\left(y_{1}-y_{0}\right)\right]\right] \sin \alpha_{m} x,
\end{aligned}
$$

при $y_{0} \leq y \leq y_{1}$

$$
\begin{aligned}
U(x, y)= & U_{0} \frac{y}{y_{1}}+\tau \sum_{m=1}^{\infty} \frac{2 \sin \alpha_{m} x_{0}}{\alpha_{m} \pi m \varepsilon_{0}} \\
& \times \frac{\operatorname{sh}\left[\alpha_{m}\left(y_{1}-y\right)\right]}{\operatorname{sh}\left[\alpha_{m} y_{1}\right]}\left(\operatorname{ch}\left[\alpha_{m} y_{0}\right]-1\right) \sin \alpha_{m} x
\end{aligned}
$$

Формулы (10), (11) позволяют вычислить постоянное значение плотности зарядов $\tau=$ const, так, чтобы нулевая эквипотенциаль проходила через заданную точку

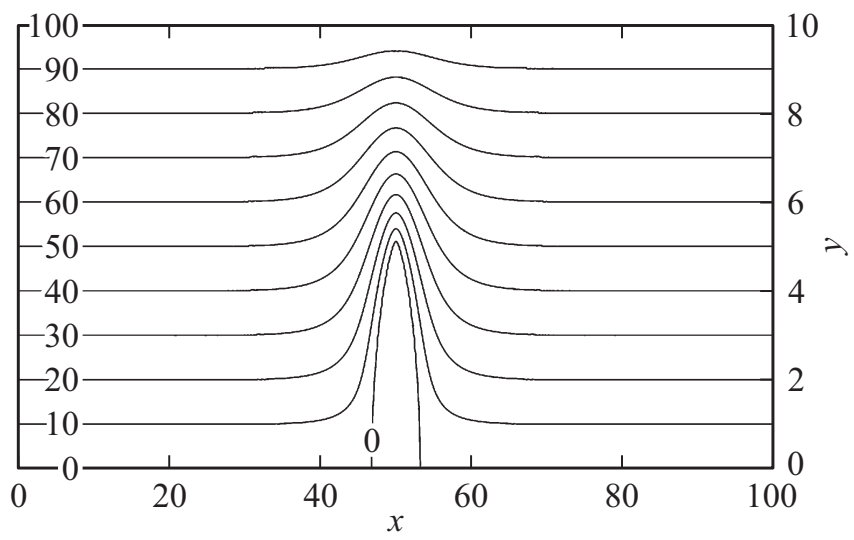

Рис. 5. Картина эквипотенциальных линий при $L=5.00005$, $\tau=-60.4784$.

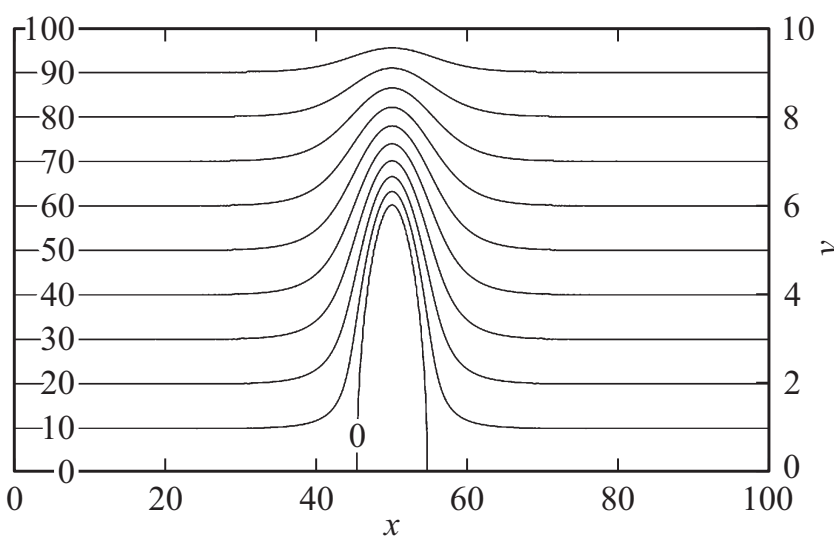

Рис. 6. Картина эквипотенциальных линий при $L=6$, $\tau=-108.21$. 
области $\left(x_{\tau}, y_{\tau}\right)$ :

$$
U\left(x_{\tau}, y_{\tau}\right)=0
$$

В частности, для заданного значения высоты $L$ полевого острия в формуле (12) координаты точки $-x_{\tau}=x_{0}$, $y_{\tau}=L$.

При численных расчетах были рассмотрены следующие значения параметров в безразмерных величинах $x_{1}=100, x_{0}=50, y_{1}=10, y_{0}=5, U_{0}=100$.

На рис. 2-4 представлены распределение электростатического потенциала и картины эквипотенциальных линий во всей области диодной системы при заданных значениях плотности зарядов $\tau$ : при $\tau=-50$ (рис. 2), при $\tau=-200$ (рис. 3), при $\tau=-500$ (рис. 4).

На рис. 5,6 представлены картины эквипотенциальных линий во всей области диодной системы при заданных значениях высоты полевого катода $L$. Из выражений $(10)-(12)$ для $L=5.00005$ плотность зарядов: $\tau=-60.4784$ (рис. 5); для $L=6$ плотность зарядов: $\tau=-108.21$ (рис. 6).

\section{Заключение}

В работе моделируется двумерная диодная система с полевым эмиттером лезвийной формы. Эмиттер расположен на плоской подложке, параллельной плоскости анода. Для того чтобы найти распределение электростатического потенциала в аналитическом виде, влияние полевого эмиттера заменяется влиянием заряженной нити. Таким образом, была поставлена граничная задача (1) для уравнения Пуассона. Для ее решения использовался метод разделения переменных в декартовых координатах. Распределение потенциала (2) найдено во всей области моделируемой системы в аналитическом виде (5)-(9). В соответствии с полученными в явном виде формулами, для конкретных значений параметров и постоянной функции плотности заряженной нити представлены графики распределения потенциала и эквипотенциальных линий. Все геометрические размеры системы и значения потенциалов на электродах представляют собой параметры задачи.

\section{Конфликт интересов}

Авторы заявляют, что у них нет конфликта интересов.

\section{Список литературы}

[1] Al-Tabbakh A.A. // Indian J. Phys. 2019. Vol. 93. N 1 P. 41-46. DOI: $10.1007 / \mathrm{s} 12648-018-1276-3$

[2] Kim J.H., Kang J.S., Park K.C. // Micromachines. 2018. Vol. 9. N 12. P. 648. DOI: $10.3390 / \mathrm{mi} 9120648$

[3] Wu M., Tafel A., Hommelhoff P., Spiecker E. // Appl. Phys. Lett. 2019. Vol. 114. N 1. P. 013101. DOI: 10.1063/1.5055227
[4] Шестеркин В.И., Соколова Т.Н., Морев С.П., Бессонов Д.А., Сурменко Е.Л., Дармаев А.Н., Комаров Д.А., муравьев Э.К., Шалаев П.Д., Шумихин К.В. // Радиотехника и электроника. 2016. Т. 61. Вып. 9. С. 896 904. DOI: https://doi.org/10.7868/S0033849416090114 [Shesterkin V.I., Sokolova T.N., Morev S.P., Bessonov D.A., Surmenko E.L., Darmaev A.N., Komarov D.A., Murav'ev E.K., Shalaev P.D. // J. Commun. Techn. Electron. 2016. Vol. 61. N 9. P. 1044-1051.

DOI: $10.1134 / \mathrm{S} 1064226916090114]$

[5] Бугаев А.С., Виноградова Е.М., Егоров Н.В., Шешин Е.П. Автоэлектронные катоды и пушки. Долгопрудный: Изд. дОм „ИНТЕЛЛЕКТ“, 2017. 288 с.

[6] Еgгоров Н.В., Шешин Е.П. Автоэлектронная эмиссия. Принципы и приборы. Долгопрудный: Изд. дом „Интеллект“, 2011. 703 с.

[7] Egorov N.V., Vinogradova E.M. // User Modeling and User-Adapted Interaction. 2003. V. 72, N 2. P. 103-111. DOI: $10.1016 / \mathrm{S} 0042-207 \mathrm{X}(03) 00107-6$

[8] Nikiforov K.A., Trofi V.V., Egorov N.V. // J. Phys.: Conf. Series. 2016. Vol. 741. N 1. P. 012009. DOI: 10.1088/17426596/741/1/012009

[9] Jityaev I.L., Svetlichnyi A.M. J. Vacuum Sci. Technol. B: Nanotechnol. Microelectron. 2019. Vol. 37. N 1. P. 012201. DOI: $10.1116 / 1.5051253$

[10] Tang W., Shiffler D., Cartwright K.L. // J. Appl. Phys. 2011. Vol. 110. N 3. P. 034905. DOI: $10.1063 / 1.3615846$

[11] Parveen S., Kumar A., Husain S., Zulfequar M., Husain M. // Phys. B: Condens. Matter. 2018. Vol. 550. P. 15-20. DOI: $10.1016 /$ j.physb.2018.08.016

[12] Давидович М.В., Яфбаров Р.К. // ЖТФ. 2018. Т. 88. Вып. 2. C. 283-293. DOI: 10.21883/JTF.2018.02.45422.2375 [Davidovich M.V., Yafarov R.K. // Tech. Phys. 2018. Vol. 63. N 2. P. 274284. DOI: $10.1134 / \mathrm{S} 106378421802010 \mathrm{X}]$

[13] Соминский Г.Г., Сезонов В.Е., Тарадаев С.П., Вдовичев С.Н. // ЖТФ. 2019. Т. 89. Вып. 1. С. 142-146. DOI: 10.21883/JTF.2019.01.46976.112-18

[14] Егоров Н.В., Антонов А.Ю., Вараюнь М.И. // Поверхность. Рентгеновские, синхротронные и нейтронные исследования. 2018. Т. 10. С. 72 79. DOI: $10.1134 / \mathrm{S} 0207352818100086$ [Egorov N.V., Antonov A.Y., Varayun' M.I. // J. Surf. Investigation. 2018. Vol. 12. N 5. P. 1005-1012. DOI: $10.1134 / \mathrm{S} 1027451018050245]$

[15] Виноградова Е.М., Егоров Н.В., Мутул М.Г., Шэнь ЧэЧоу. // ЖТФ. 2010. Т. 80. Вып. 5. С. 1-4. [Vinogradova E.M., Egorov N.V., Mutul M.G., Shen C.-C. // Tech. Phys. 2010. T. 55. N 5. P. 591-594. DOI: 10.1134/S1063784210050014]

[16] Sorokina V., Nikiforov K. // Young Researchers in Vacuum Micro/Nano Electronics. VMNE-YR. 2016. Proceedings 7880415. DOI: 10.1109/VMNEYR.2016.7880415

[17] Виноградова Е.М., Егоров Н.В., Телевный Д.С. // ЖТФ. 2014. Т. 84. Вып. 2. С. 139-144. [Vinogradova E.M., Egorov N.V., Televnyi D.S. // Tech. Phys. 2014. T. 59. N 2. P. 291-296. DOI: 10.1134/S1063784214020236]

[18] Виноградова Е.М., Егоров Н.В., Климаков А.А.. // ЖТФ. 2015. Т. 85. Вып. 2. С. 20-23. [Vinogradova E.M., Egorov N.V., Klimakov A.A. // Tech. Phys. 2015. T. 60. N 2. P. 176-179. DOI: 10.1134/S1063784215020243]

[19] Vinogradova E.M., Egorov N.V., Televnyy D.S. // Vacuum. 2016. Vol. 127. P. 45-50. DOI: 10.1016/j.vacuum.2016.01.026 